\title{
Biocompatible Polymeric Microparticles Produced by a Simple Biomimetic Approach
}

\author{
Ana M. S. Costa, Manuel Alatorre-Meda,* Nuno M. Oliveira, and João F. Mano* \\ 3B's Research Group-Biomaterials, Biodegradables and Biomimetics, University of Minho, Headquarters of the European Institute \\ of Excellence on Tissue Engineering and Regenerative Medicine, AvePark, Zona Industrial da Gandra, S. Claudio do Barco, 4806-909 \\ Caldas das Taipas, Guimarães, Portugal and ICVS/3B's-PT Government Associate Laboratory, Braga/Guimarães, Portugal
}

\section{Supporting Information}

\begin{abstract}
The use of superhydrophobic surfaces to produce polymeric particles proves to be biologically friendly since it entails the pipetting and subsequent cross-linking of polymeric solutions under mild experimental conditions. Moreover, it renders encapsulation efficiencies of $\sim 100 \%$. However, the obtained particles are 1 to $2 \mathrm{~mm}$ in size, hindering to a large extent their application in clinical trials. Improving on this technique, we propose the fabrication of polymeric microparticles by spraying a hydrogel precursor over superhydrophobic surfaces followed by photo-cross-linking. The particles were produced from methacrylamide chitosan (MA-CH) and characterized in terms of their size and morphology. As demonstrated by optical and fluorescence microscopy, spraying followed by photo-cross-linking led, for the first time, to the production of spherical particles with diameters on the order of micrometers, nominal sizes not attainable by pipetting. Particles such as these are suitable for medical applications such as drug delivery and tissue engineering.
\end{abstract}

\section{INTRODUCTION}

Polymeric particles for biomedical applications can be produced by a plethora of different routes, including emulsion polymerization, solvent evaporation/extraction, and phase separation (coacervation), among others, all of which are indeed effective for their massive production but might also present major limitations related to the use of organic/toxic solvents or high temperature, compromising the encapsulation of certain bioactive materials. ${ }^{1,2}$ Recognizing the aforementioned drawbacks of conventional protocols, our group has recently proposed the implementation of biomimetic synthesis procedures based on the utilization of superhydrophobic surfaces for the production of polymeric mono- and multilayered particles, ${ }^{3,4}$ also inspiring the work of others. ${ }^{5}$ The strategy, encompassing the pipetting and subsequent crosslinking of polymeric solutions containing the drug to be released, is rather simple and reproducible, allowing the production of totally biocompatible materials with encapsulation efficiencies of $\sim 100 \%$. However, the minimum size and shape of produced particles are strongly dependent on the solution viscosity/volume and substrate conditions, resulting in the production of particles not smaller than $1 \mathrm{~mm}$ in diameter, hampering in consequence their tentative use in clinical trials.

Improving on this biomimetic technique, here we demonstrate for the first time the production of polymeric microparticles on top of superhydrophobic surfaces. The particles were produced from aqueous solutions of $\mathrm{MA}-\mathrm{CH}$ by spraying followed by photo-cross-linking. On the one hand, $\mathrm{MA}-\mathrm{CH}$ is anticipated to endow the particles with the favorable biological properties of $\mathrm{CH}$ such as its outstanding biocompat- ibility $^{6-8}$ and biodegradability ${ }^{9,10}$ while being soluble in water and presenting methacrylic groups necessary for cross-linking. ${ }^{11}$ On the other hand, we prove that spraying onto superhydrophobic surfaces is conducive to the production of increasingly smaller particles with nominal diameters on the order of micrometers.

\section{MATERIALS AND METHODS}

2.1. Materials. Glass microscope slides were from Medline (Spain). Acetic acid was from VWR (Belgium). Tetraethyl orthosilicate (TEOS, 98\%), methacrylic anhydride (MA, $\geq 92 \%$ ), ammonium hydroxide solution (30-33\%), 2-hydroxy-4'-(2-hydroxyethoxy)-2-methylpropiophenone (I2959, 98\%), and $1 \mathrm{H}, 1 \mathrm{H}, 2 \mathrm{H}, 2 \mathrm{H}$ perfluorodecyltriethoxysilane (silane, 97\%) were from Sigma-Aldrich. Chitosan 95/20 was from Heppe Medical Chitosan GmbH (Germany). $\mathrm{KBr}$ powder was from PIKE Technologies (USA). Deuterium oxide was from Cambridge Isotope Laboratories (USA). All materials were used as received. Unless otherwise stated, water purified in a $18 \mathrm{M} \Omega \mathrm{cm}$ Milli-Q Plus water system was used throughout.

2.2. Synthesis and Characterization of Superhydrophobic Surfaces and MA-CH. The reader is referred to the Supporting Information.

2.3. Synthesis and Characterization of MA-CH Microparticles. On the day of the experiment, $2.5 \mathrm{w} / \mathrm{v} \% \mathrm{MA}-\mathrm{CH}$ was dissolved in water containing $0.25 \mathrm{w} / \mathrm{v} \% \mathrm{I} 2959$. To produce the MA$\mathrm{CH}$ microspheres, we dispensed this solution with a generic sprayer (nozzle sectional area $=7.55 \times 10^{-4} \mathrm{~cm}^{2}$, input pressure $\sim 400 \mathrm{mbar}$;

Received: January 24, 2014

Revised: April 9, 2014

Published: April 10, 2014 


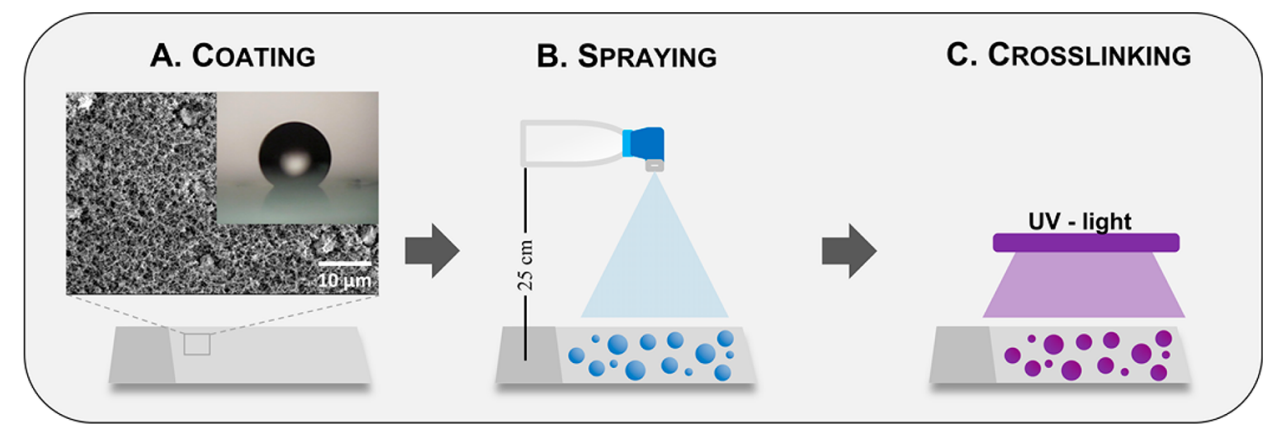

Figure 1. Scheme depicting MA-CH particle production. (A) Microscope glass slides were coated with a carbon/silica/silane network in order to obtain a superhydrophobic surface. WCA and SEM images of the surface structure are shown. (B) MA-CH solution was dispensed using a generic sprayer placed $25 \mathrm{~cm}$ above the superhydrophobic surface. (C) MA-CH particles were cross-linked using UV light for 1 min in the presence of I2959.

Before Crosslinking

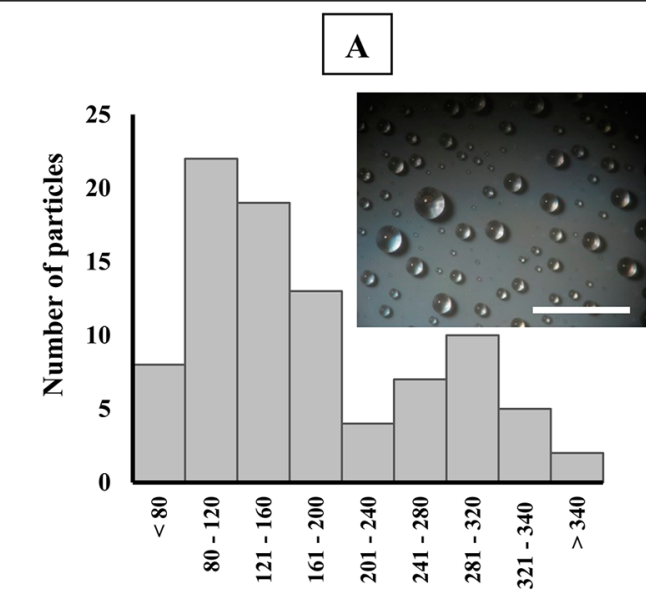

Particles diameter $[\mu \mathrm{m}]$

\section{After Crosslinking}

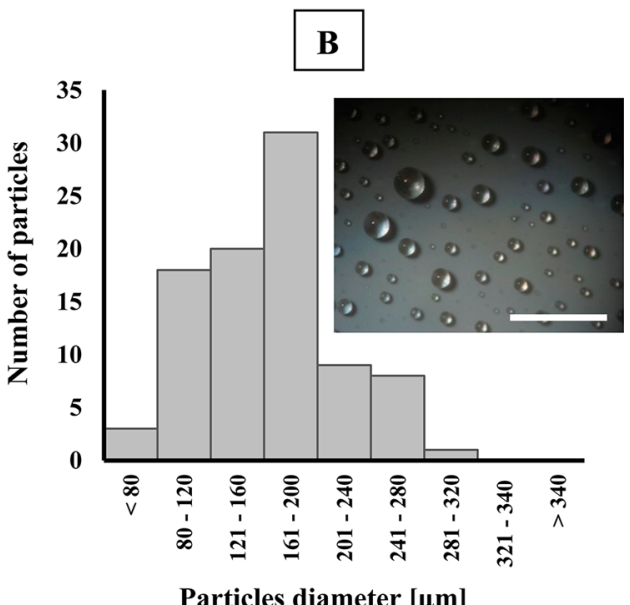

Particles diameter $[\mu \mathrm{m}]$
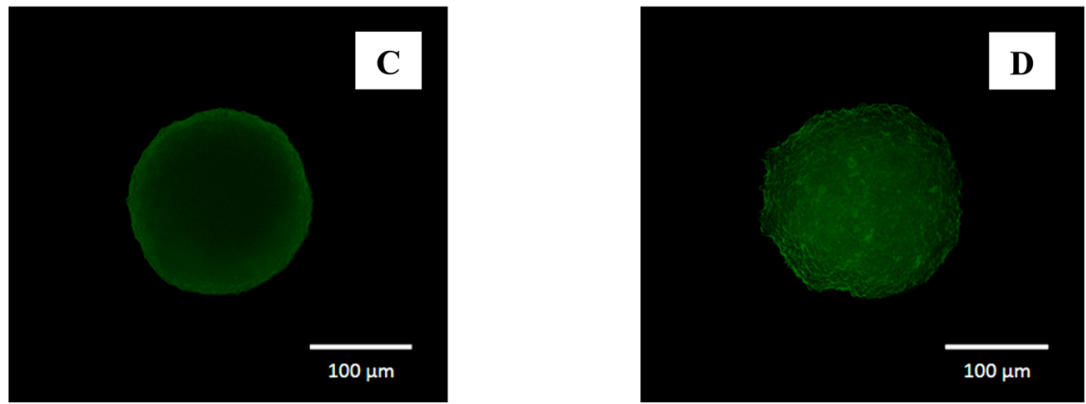

Figure 2. Characterization of MA-CH particles before and after cross-linking by optical (A and B) and fluorescence microscopy (C and D). Size distribution histograms in panels A and B were calculated from the measurement of the mean diameter (parallel to the surface) of imaged particles. The scale bar in optical micrographs of panels A and B stands for $1 \mathrm{~mm}$.

see the Supporting Information) by spraying once $25 \mathrm{~cm}$ above the superhydrophobic surface (see Figure 1). Afterward, the obtained polymeric droplets were cross-linked using UV light (365 nm, $11.4 \mathrm{~W} /$ $\mathrm{cm}^{2}$ ) for $1 \mathrm{~min}$. The sprayed droplets were observed by optical (TR500; VWR) and fluorescence microscopy (Axio Imager Z1m; Zeiss) before and after cross-linking. The mean particle size (diameter) and shape factor (SF, ratio between diameters of particles orthogonal and parallel to the surface) of 90 different particles were obtained from 14 independent optical images by using ImageJ (see the Supporting Information). For fluorescence microscopy, images were recorded in the green channel $(505 \mathrm{~nm})$ and analyzed using the ZEN software supplied with the instrument.

2.4. Statistical Analysis. Statistical significance was considered at a probability of $p<0.05$ using the $t$ test.

\section{RESULTS AND DISCUSSION}

3.1. General Overview of the Proposed Technology. Polymeric microparticles were produced for the first time on top of superhydrophobic surfaces. The microparticles were produced from MA-CH, a well-known biopolymer, and cross- 
linked upon light irradiation in the presence of I2959, a cytocompatible cross-linker. ${ }^{12,13}$ The obtained particles were characterized for their cross-linking degree, weight, size, and morphology before and after cross-linking in order to assess the feasibility of the proposed method.

The first step in our study was devoted to finding the optimal experimental conditions, in particular, those relative to the MA$\mathrm{CH}$ concentration, cross-linking time, spraying height for the chosen sprayer, and particle recovery. With respect to the MA$\mathrm{CH}$ concentration and cross-linking time, preliminary studies with particles produced by pipetting demonstrated that polymer concentrations below $1 \%$ gave rise to liquidlike gels even after cross-linking times greater than $10 \mathrm{~min}(365 \mathrm{~nm}$, $11.4 \mathrm{~W} / \mathrm{cm}^{2}$ ). By contrast, when the concentration of $\mathrm{MA}-\mathrm{CH}$ was increased to ca. $3 \%$, the gels were found to adopt a more solid structure at cross-linking times of around $1 \mathrm{~min}$. As a result, the $\mathrm{MA}-\mathrm{CH}$ concentration and cross-linking time were chosen to be $2.5 \%$ and $1 \mathrm{~min}$, respectively (the selected concentration was the highest one allowing a free flow of the polymeric solution through the sprayer nozzle). Regarding the spraying height, it was decided to amount to $25 \mathrm{~cm}$ above the surface since shorter heights $(\mathrm{ca} .5-10 \mathrm{~cm}$ ) rendered particles equivalent to those obtained by pipetting, easily seen by the naked eye, and higher ones (ca. $30-40 \mathrm{~cm}$ ) reduced the quantity of dispensed solution given the restricted area of the surfaces (as observed by microscopy, images not shown). Finally, concerning particle recovery, we concluded that the best way to remove the particles from the surfaces was by mechanical methods such as by a slight tapping by the noncoated face of the surfaces or by direct motion with the help of a needle, not requiring any kind of purification steps. In this context, we also verified that the removal of particles is facilitated while working under humidified-air conditions so that the particles remain hydrated. The chosen experimental conditions made possible the administration of ca. $17 \pm 1 \mathrm{mg}$ of polymeric solution per cycle $(n=6)$, resulting in a final weight of particles after cross-linking of $13 \pm 2 \mathrm{mg}(n=6)$ (that is, a shrinking ratio of $23.5 \%)$. Inferred from optical micrographs of the surfaces after particle recovery, the process yield was determined to be ca. $100 \%$ inasmuch as almost no particle was observed all along the inspected surfaces (images not shown).

3.2. Particle Characterization by Optical and Fluorescence Microscopy. Figure 2 presents optical and fluorescence micrographs of the produced microparticles before and after cross-linking. Regarding the optical characterization, panel A shows that dispensed particles (before cross-linking) were distributed all along the sprayed substrates with a welldefined spherical shape, although presenting a rather broad size distribution spanning from ca. 80 to $400 \mu \mathrm{m}$. The spherical shape $(\mathrm{SF}=0.92 \pm 0.06)$ is promoted by the topography of the surfaces described in the Supporting Information (see Figure S1). It is well accepted that rough (porous) surfaces prompt the formation of a solid-air interface by the inner parts of asperities. Then, when water droplets are dispensed, they organize preferentially by this interface, reducing to a high extent the solid-liquid contact area and therefore liquid adhesion. Referred to as the Cassie-Baxter state, ${ }^{14}$ this regime proves to preserve the spherical shape of droplets as long as their diameters are larger than the pitch between asperities and no external forces such as pressure are applied. ${ }^{15,16}$ Thus, the spherical shape of our particles is a consequence of the observed pitch between asperities (Figure S1B), which was found to amount to ca. $2 \mu \mathrm{m}$, a characteristic length at least 1 order of magnitude shorter than the diameter of the smallest sprayed droplets. Interestingly, this outlined sphericity of the sprayed particles, combined with their small dimensions, proved to guarantee a lower surface interaction with the superhydrophobic substrates. As shown in Table 1, the fractional contact area (FA) of microparticles was determined to be ca. 4 times smaller than that of particles dispensed by pipetting.

Table 1. Surface Contact Area (SA) and Fractional Area in Contact with the Substrates (FA) for Microdroplets and Macrodroplets $^{a}$

$\begin{array}{ccc} & \text { SA }\left(\mathrm{mm}^{2}\right) & \text { FA (\%) } \\ \text { microdroplet }(R \cong 0.1 \mathrm{~mm}) & 5.8 \times 10^{-4} & 0.4 \\ \text { macrodroplet }(R \cong 1 \mathrm{~mm}) & 1.9 \times 10^{-1} & 1.7\end{array}$

${ }^{a} R$ stands for the particle radius. For details on these calculations, the reader is referred to the Supporting Information.

On the other hand, regarding the broad size distribution of sprayed particles $(80-400 \mu \mathrm{m})$, we speculate it to result from droplet fragmentation, namely, the formation of smaller droplets from larger ones due to their high-speed collision onto solid surfaces during spraying. ${ }^{17}$ This is likely to occur considering the already-cited simplicity of the experimental setup (section 2.3) by which physical variables such as spraying pressure and nozzle dimensions were not varied. At this point, it is noteworthy that other techniques arguably avoid fragmentation by spraying directly onto liquid media. ${ }^{18}$ Yet, they show the major drawback of requiring additional washing steps devoted to the removal of residual counterions from the bulk (originally present in excess for cross-linking), impacting to a high extent the process yield and not guaranteeing $100 \%$ encapsulation. ${ }^{18,19}$ As mentioned before, this is not a problem in the current protocol. In our case, the mere implementation of more sophisticated sprayers enabling the tuning of the aforementioned variables has proved to be conducive to achieving better control of the size distribution of the droplets (i.e., better control of fragmentation; results to be published).

With respect to particle evolution after cross-linking, panel B depicts two features worth stressing. First, the particles preserved the well-defined spherical geometry exhibited before cross-linking ( $\mathrm{SF}=0.93 \pm 0.08$ ), highlighting the advantages of employing a noninvasive procedure for particles processing. ${ }^{1}$ Second, compared to sprayed particles, the width of the size distribution was slightly shortened to the $80-300 \mu \mathrm{m}$ interval, which can fairly be attributed to water expulsion from the crosslinked polymeric core and the partial dehydration of free water from the surface of already-formed hydrogels. ${ }^{20,21}$

Taking advantage of the natural fluorescence of $\mathrm{CH}^{22}$ fluorescence microscopy experiments were also carried out in order to gain insight into the inner structure of the produced particles, as shown in panels $\mathrm{C}$ and $\mathrm{D}$. These panels confirm the spherical geometry and size of the particles revealed by optical microscopy; however, they also reveal clear differences in the fluorescence emission from samples before (panel C) and after cross-linking (panel D). Panel C displays the imaged particle as having a well-defined contour but a faint core; meanwhile, panel D shows a particle with a well-defined core structure, reflecting a homogeneous distribution of fluorescence emission points. Regarding panel $\mathrm{C}$, the observed blurred profile suggests the presence of a comparatively higher quantity of water, 
distorting the fluorescence emission of $\mathrm{CH}$. Technically speaking, such a distortion can result from background emission, spherical aberration, and/or a combination of both. $^{23,24}$ Concerning panel $\mathrm{D}$, on the other hand, the observed, more defined profile provides evidence of (i) the confinement of $\mathrm{CH}$ chains to a sound spatial restructuring because of cross-linking, ${ }^{25}$ leading to an enhanced intensity of the in-focus fluorescence related to the accumulation of emission points within a restricted area, ${ }^{23}$ and (ii) water expulsion from the particle core and the eventual evaporation of superficial water (as also inferred by optical microscopy), ${ }^{20,21}$ diminishing to a high extent (not to say avoiding) the appearance of the aforementioned image artifacts. Interestingly and highlighting our particles as suitable vehicles for drug delivery, NMR results demonstrated that the practiced conditions led to a $76.3 \%$ conversion of methacrylic groups, providing evidence of the right cross-linking of $\mathrm{CH}$ chains (see the Supporting Information). As widely cited in literature, the spatial restructuring of $\mathrm{CH}$ chains within the particles after cross-linking constitutes the driving mechanism by which therapeutic molecules can be encapsulated. ${ }^{26}$ Such reorganization is mandatory not only for the initial protection of encapsulated cargos but also for their subsequent controlled release. $^{1}$

Taken together, all of these observations ratify that despite the simplicity of the spray system used, which is in part responsible for the obtained size distribution, this method proves to be suitable for obtaining polymeric particles with sizes ca. 1 order of magnitude smaller with respect to the current methodology, namely, by pipetting, ${ }^{3,4}$ reaching a high process yield of around $100 \%$. Importantly, contrary to the wetchemistry procedures commonly employed to produce polymeric microparticles ${ }^{1}$ our proposed protocol is also demonstrated to be biologically friendly, straightforward, and cost-effective. In fact, we anticipate that the process can be scalable by employing surfaces with larger sectional areas and also by optimizing the experimental conditions for more robust sprayers operating at varying spraying pressures, with different nozzles, and allowing, in consequence, to achieve tunable flow rates. Moreover, the simplicity of the concept and the attainable amphiphobic character of the surfaces ${ }^{27}$ make it possible to expand this technology to other purposes such as cosmetics, agriculture, and electronics, where solvents different than water are often required. On the other hand, regarding the particles per se, the biocompatible properties of $\mathrm{CH}$ combined with the mild processing conditions point them out as suitable microcarriers for many bioactive molecules such as cells and proteins, which is beneficial for different fields such as biology and tissue engineering. ${ }^{28,29}$

\section{OUTLOOK}

This work proposes a new strategy to produce polymeric particles based on the use of superhydrophobic surfaces and spraying. Compared to other methodologies, the sketched approach proved to be simple, fast, cost-effective, and totally biocompatible, allowing the production of polymeric microparticles under mild experimental conditions without using any kind of harsh materials. As such, the proposed strategy is anticipated to constitute a new, versatile tool for producing microcarriers for both science and technology. It has to be noted, however, that the possibility of producing particles with increasingly smaller dimensions and narrower size distributions will rely directly on the optimization of the present process and/or the use of more robust dispensing systems. As previously mentioned, the process can be scalable by employing superhydrophobic surfaces with larger sectional areas.

\section{ASSOCIATED CONTENT}

S Supporting Information

Synthesis and characterization of both superhydrophobic surfaces and MA-CH. Characterization of cross-linking degree by NMR. Mechanical characterization of the sprayer used. This material is available free of charge via the Internet at http:// pubs.acs.org.

\section{AUTHOR INFORMATION}

\section{Corresponding Authors}

*E-mail: manuel_alatorre@yahoo.com.mx.

*E-mail: jmano@dep.uminho.pt.

\section{Notes}

The authors declare no competing financial interest.

\section{ACKNOWLEDGMENTS}

We thank Ivo Aroso and Ana Isabel Neto for their valuable support with FTIR and compression experiments, respectively. A.M.S.C. thanks FCT for financial support through grant BIM/ PTDC/CTM-BPC/112774/2009_02. M.A.-M. thanks CONACyT (Mexico) for financial support through post-doc grant no. 203732. N.M.O. thanks FCT for financial support through Ph.D. scholarship no. SFRH/BD/73172/2010. This work was funded by the European Union's Seventh Framework Programme (FP7/2007-2013) under grant agreement no. REGPOT-CT2012-316331-POLARIS, by FEDER through the Competitive Factors Operation Program--COMPETE, and by national funds through FCT - Fundação para a Ciência e a Tecnologia in the scope of project PTDC/CTM-BIO/ $1814 / 2012$.

\section{REFERENCES}

(1) Varde, N. K.; Pack, D. W. Microspheres for controlled release drug delivery. Expert Opin. Biol. Ther. 2004, 4 (1), 35-51.

(2) Lima, A. C.; Sher, P.; Mano, J. F. Production methodologies of polymeric and hydrogel particles for drug delivery applications. Expert Opin. Drug Delivery 2012, 9 (2), 231-248.

(3) Song, W. L.; Lima, A. C.; Mano, J. F. Bioinspired methodology to fabricate hydrogel spheres for multi-applications using superhydrophobic substrates. Soft Matter 2010, 6 (23), 5868-5871.

(4) Lima, A. C.; Custódio, C. A.; Alvarez-Lorenzo, C.; Mano, J. F. Biomimetic Methodology to Produce Polymeric Multilayered Particles for Biotechnological and Biomedical Applications. Small 2013, 9 (15), 2487-2492.

(5) Deng, X.; Paven, M.; Papadopoulos, P.; Ye, M.; Wu, S.; Schuster, T.; Klapper, M.; Vollmer, D.; Butt, H.-J. Solvent-Free Synthesis of Microparticles on Superamphiphobic Surfaces. Angew. Chem., Int. Ed. 2013, 52 (43), 11286-11289.

(6) Rodríguez-Velázquez, E.; Silva, M.; Taboada, P.; Mano, J. F.; Suárez-Quintanilla, D.; Alatorre-Meda, M. Enhanced Cell Affinity of Chitosan Membranes Mediated by Superficial Cross-Linking: A Straightforward Method Attainable by Standard Laboratory Procedures. Biomacromolecules 2014, 15 (1), 291-301.

(7) Alatorre-Meda, M.; Taboada, P.; Hardl, F.; Wagner, T.; Freis, M.; Rodriguez, J. R. The influence of chitosan valence on the complexation and transfection of DNA: The weaker the DNA-chitosan binding the higher the transfection efficiency. Colloids Surf., B 2011, 82 (1), 5462

(8) Alves, N. M.; Mano, J. F. Chitosan derivatives obtained by chemical modifications for biomedical and environmental applications. Int. J. Biol. Macromol. 2008, 43 (5), 401-414. 
(9) Krajewska, B.; Wydro, P.; Jańczyk, A. Probing the Modes of Antibacterial Activity of Chitosan. Effects of $\mathrm{pH}$ and Molecular Weight on Chitosan Interactions with Membrane Lipids in Langmuir Films. Biomacromolecules 2011, 12 (11), 4144-4152.

(10) Krajewska, B.; Kyziol, A.; Wydro, P. Chitosan as a subphase disturbant of membrane lipid monolayers. The effect of temperature at varying pH: II. DPPC and cholesterol. Colloids Surf., A 2013, 434 (0), 359-364.

(11) Yu, L. M. Y.; Kazazian, K.; Shoichet, M. S. Peptide surface modification of methacrylamide chitosan for neural tissue engineering applications. J. Biomed. Mater. Res., Part A 2007, 82A (1), 243-255.

(12) Mironi-Harpaz, I.; Wang, D. Y.; Venkatraman, S.; Seliktar, D. Photopolymerization of cell-encapsulating hydrogels: Crosslinking efficiency versus cytotoxicity. Acta Biomater. 2012, 8 (5), 1838-1848.

(13) Jeon, O.; Bouhadir, K. H.; Mansour, J. M.; Alsberg, E. Photocrosslinked alginate hydrogels with tunable biodegradation rates and mechanical properties. Biomaterials 2009, 30 (14), 2724-2734.

(14) Cassie, A. B. D.; Baxter, S. Wettability of porous surfaces. Trans. Faraday Soc. 1944, 40, 0546-0550.

(15) Jung, Y. C.; Bhushan, B. Wetting behaviour during evaporation and condensation of water microdroplets on superhydrophobic patterned surfaces. J. Microsc. (Oxford, U.K.) 2008, 229 (1), 127-140.

(16) Wenzel, R. N. Surface roughness and contact angle. J. Phys. Colloid Chem. 1949, 53 (9), 1466-1467.

(17) PasandidehFard, M.; Qiao, Y. M.; Chandra, S.; Mostaghimi, J. Capillary effects during droplet impact on a solid surface. Phys. Fluids 1996, 8 (3), 650-659.

(18) Suksamran, T.; Opanasopit, P.; Rojanarata, T.; Ngawhirunpat, T.; Ruktanonchai, U.; Supaphol, P. Biodegradable alginate microparticles developed by electrohydrodynamic spraying techniques for oral delivery of protein. J. Microencapsulation 2009, 26 (7), 563-570.

(19) Ramadan, Q.; Gijs, M. A. M. Simultaneous magnetic particles washing and concentration in a microfluidic channel. Procedia Chem. 2009, 1 (1), 1499-1502.

(20) Fondecave, R; Wyart, F. B. Wetting laws for polymer solutions. Europhys. Lett. 1997, 37 (2), 115-120.

(21) Yoshida, H.; Hatakeyama, T.; Hatakeyama, H. Characterization of water in polysaccharide hydrogels by DSC. J. Therm. Anal. 1993, 40 (2), 483-489.

(22) Bhaskar, S.; Gibson, C. T.; Yoshida, M.; Nandivada, H.; Deng, X. P.; Voelcker, N. H.; Lahann, J. Engineering, Characterization and Directional Self-Assembly of Anisotropically Modified Nanocolloids. Small 2011, 7 (6), 812-819.

(23) Waters, J. C. Accuracy and precision in quantitative fluorescence microscopy. J. Cell Biol. 2009, 185 (7), 1135-1148.

(24) Egner, A.; Hell, S. Aberrations in Confocal and Multi-Photon Fluorescence Microscopy Induced by Refractive Index Mismatch. In Handbook of Biological Confocal Microscopy; Pawley, J. B., Ed.; Springer: New York, 2006; pp 404-413.

(25) Berger, J.; Reist, M.; Mayer, J. M.; Felt, O.; Peppas, N. A.; Gurny, R. Structure and interactions in covalently and ionically crosslinked chitosan hydrogels for biomedical applications. Eur. J. Pharm. Biopharm. 2004, 57 (1), 19-34.

(26) Al-Qadi, S.; Alatorre-Meda, M.; Zaghloul, E. M.; Taboada, P.; Remunan-Lopez, C. Chitosan-hyaluronic acid nanoparticles for gene silencing: The role of hyaluronic acid on the nanoparticles' formation and activity. Colloids Surf., Bs 2013, 103, 615-623.

(27) Deng, X.; Mammen, L.; Butt, H.-J.; Vollmer, D. Candle Soot as a Template for a Transparent Robust Superamphiphobic Coating. Science 2012, 335 (6064), 67-70.

(28) Croisier, F.; Jérôme, C. Chitosan-based biomaterials for tissue engineering. Eur. Polym. J. 2013, 49 (4), 780-792.

(29) Oliveira, M. B.; Mano, J. F. Polymer-Based Microparticles in Tissue Engineering and Regenerative Medicine. Biotechnol. Prog. 2011, 27 (4), 897-912. 